\title{
Neuroprotective effect of berberine agonist against impairment of learning and memory skills in severe traumatic brain injury via Sirt1/p38 MAPK expression
}

\author{
JIANPING WANG $^{1}$ and YAMIN ZHANG $^{2}$ \\ Departments of ${ }^{1}$ Emergency and ${ }^{2}$ Neurological Rehabilitation, Gansu Provincial Hospital, \\ Lanzhou, Gansu 730000, P.R. China
}

Received January 22, 2017; Accepted September 20, 2017

DOI: $10.3892 / \mathrm{mmr} .2018 .8674$

\begin{abstract}
Berberine has multiple clinical applications, including the treatment of tumors, diabetes, cardiovascular diseases, hyperlipidemia, inflammation, bacterial and viral infections, cerebral ischemic injuries, mental diseases, Alzheimer's disease and osteoporosis. In the present study, the neuroprotective effect of berberine agonist rescue learning and memory in severe traumatic brain injury (TBI), and the possible mechanism underlying these observations was explored. The protective effect of berberine agonist significantly recovered learning and memory skills, attenuated brain edema and inhibited matrix metalloproteinase- 3 and -9 protein expression in mice with severe TBI. Berberine agonist significantly reduced inflammation, oxidative stress and apoptosis levels in mice with severe TBI. Berberine agonist promoted choline acetyltransferase activity and inhibited the activity of acetylcholinesterase. Collectively, results of the present study revealed that the neuroprotective effect of berberine agonist rescues learning and memory skills in severe TBI.
\end{abstract}

\section{Introduction}

Traumatic brain injury (TBI) is a prevalent disease in modern society. The fatality and disability rates of TBI are the highest among traumas of human organs (1). The incidence of TBI in USA is $2 \%$, and 500,000 new TBI cases are recorded annually, including $10 \%$ severe TBI, which leads to 20,000 mortality and 30,000 disability cases, resulting in significant direct and indirect economic losses (2). With the rapid development of the global economy and modern transportation, TBI has become

Correspondence to: Dr Jianping Wang, Department of Emergency, Gansu Provincial Hospital, 204 Donggang West Road, Lanzhou, Gansu 730000, P.R. China

E-mail: wzekyr2926206@126.com

Key words: berberine, severe traumatic brain injury, learning and memory, p38 mitogen-activated protein kinase an important human health issue (1). Therefore, research groups are attempting to alleviate TBI-induced neurological impairment, and improve the effectiveness of the treatment of TBI (3). TBI and the resulting pathological condition are major factors endangering human health. Sequelae of TBI include post-TBI learning and memory skill impairment, which influence the quality of life of patients (4).

TBI-induced neural death is the most prevalent cause of death in patients. Inhibition of post-injury cell death and preservation of neurons is the research focus of TBI (5). Inhibition of the cell death pathway and fundamental reduction of neuronal death is a potential treatment approach (6). Cell death can be classified into two categories, cell apoptosis and cell necrosis. Apoptosis is programmed cell death regulated by gene expression, while necrosis represents an unregulated and irreversible passive cell death (7).

Numerous biologically active natural products isolated from Chinese medical herbs frequently demonstrate physiological functions. An appropriate structural modification of these herbs may lead to the development of novel drugs with high activity and specificity (8). Drug research and development in China should focus on carrying out innovative drug research based on biologically active natural products native to China (9). Many biologically active natural products extracted from Chinese herbal medicine, including berberine, have been extensively applied in medical practice. Berberine (Fig. 1) is an isoquinoline alkaloid derived from Coptis chinensis, a plant species native to China (9). Berberine and its derivatives demonstrate multiple pharmacological effects on tumor treatment, diabetes, cardiovascular disease, hyperlipidemia, inflammation, bacterial and viral infection, cerebral ischemic injury, mental disease, Alzheimer's disease and osteoporosis (10). The aim of the present study was to determine the neuroprotective effect of berberine on learning and memory skills in severe TBI, and to explore the underlying molecular mechanism.

\section{Materials and methods}

Animals and treatment groups. Male C57BL/6J mice (6-7 weeks old; weight, 20-22 g) were obtained from the National Laboratory Animal Center of Gansu Medical College (Lanzhou, China). 
All animals were randomly allocated into one of three groups: i) Control; ii) TBI model; and iii) berberine agonist treatment. Mice were anesthetized with an intraperitoneal injection (I.I) of sodium pentobarbital (65 mg/kg; Sigma-Aldrich; Merck KGaA, Darmstadt, Germany), and $5 \mathrm{~mm}$ craniotomy was performed at the right parietal cortex. Controlled cortical impact was induced using a pneumatic piston with a $2.5 \mathrm{~mm}$ rounded metal tip. The bone flap was replaced and sealed, and the scalp was sterilized with iodophor (Sangon Biotech Co., Ltd., Shanghai, China) and sutured. Temperature of the heated cage was maintained at $37.0 \pm 0.5^{\circ} \mathrm{C}$ using a heated pad. Following injury, mice were administered $10 \mathrm{mg} / \mathrm{kg} /$ day berberine (Sigma-Aldrich; Merck KGaA) via intraperitoneal injection for 4 weeks. The present study was approved by the Ethics Committee of Gansu Provincial Hospital (Gansu, China).

Morris water maze test. Following treatment with berberine agonist, mice were subjected to a Morris water maze test (Gansu Medical College, Gansu, China; 1x0.5x0.4 m; 23 $\pm 2^{\circ} \mathrm{C}$ ). Mice learned how to navigate the maze for 4 days, and then the probe trial was removed. The time it took for mice to pass through the maze and find a platform was recorded and called the escape latency.

Measurement of inflammation and oxidation. Peripheral blood was collected following treatment with berberine agonist and centrifuged at 2,000 $\mathrm{xg}$ for $10 \mathrm{~min}$ at $4^{\circ} \mathrm{C}$. ELISA for glutathione peroxidase 1 (GSH-PX; S0055), glutathione synthetase (GSH; S0053), superoxide dismutase (SOD; S0109), malondialdehyde (MDA; S0131), tumor necrosis factor- $\alpha$ (TNF- $\alpha$; PT512) and interleukin (IL)-6 (PI326) levels was performed using commercially available kits (Beyotime Institute of Biotechnology). Choline acetyltransferase (ChAT) and acetylcholinesterase (AchE) levels were performed using commercially available kits (A079-1 and A024 respectively; Nanjing Jiancheng Biology Engineering Institute, Nanjing, China).

Western blotting. Protein homogenates were prepared from hippocampal tissues using a radioimmunoprecipitation assay (RIPA; Beyotime Institute of Biotechnology), and centrifuged at $12,000 \mathrm{x} \mathrm{g}$ for $10 \mathrm{~min}$ at $4^{\circ} \mathrm{C}$. Protein content was measured using a bicinchoninic acid assay. Proteins (50 $\mu \mathrm{g} /$ per lane) were separated by $8-10 \%$ SDS-PAGE and transferred to a polyvinylidene difluoride membrane. The membrane was blocked using $5 \%$ skimmed milk in TBS with Tween-20 and incubated with matrix metalloproteinase (MMP)-3 (sc-374029; 1:300), MMP-9 (sc-10737; 1:300), caspase-3 (sc-98785; 1:500), sirtuin 1 (Sirt1; sc-15404; 1:500), phosphorylated (p)-p38 (sc-101759; 1:500) and GAPDH (sc-25778; 1:500; all Santa Cruz Biotechnology, Inc., Dallas, TX, USA) primary antibodies at $4^{\circ} \mathrm{C}$ overnight. Following washing with TBST for $15 \mathrm{~min}$, membranes were incubated with a horseradish peroxidase-linked anti-rabbit or anti-mouse secondary antibody (sc-2004 or sc-2020; 1:2,000; Santa Cruz Biotechnology, Inc.) for $2 \mathrm{~h}$ at $37^{\circ} \mathrm{C}$ and detected using Pierce enhanced chemifluorescent western blotting substrate (cat. no. 321109; Thermo Fisher Scientific, Inc., Waltham, MA, USA).

Statistical analysis. Data are presented as the mean \pm standard deviation using SPSS version 17.0 (SPSS, Inc., Chicago,

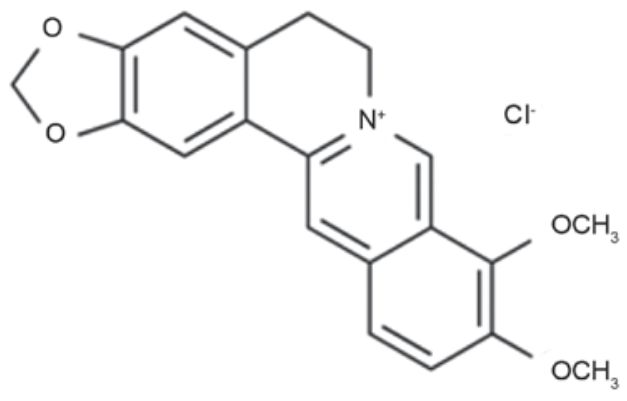

Figure 1. Chemical structure of berberine.

IL, USA). One-way or two-way analysis of variance followed by Bonferroni's post hoc test was used for multiple group comparisons. $\mathrm{P}<0.05$ was considered to indicate a statistically significant difference.

\section{Results}

Protective effect of berberine agonist evidenced by Morris water maze test. Berberine (Fig. 1) is an isoquinoline alkaloid derived from Coptis chinensis, a plant species native to China (9). To investigate whether the protective effect of berberine agonist rescues learning and memory skills in severe TBI, TBI mice were treated with berberine agonist. As presented in Fig. 2A and B, a significant increase in escape latency and a longer mean path length were observed in the TBI model group compared with the control group. The time spent in the target quadrant and the number of times mice crossed the platform was decreased in the TBI model group compared with the control group (Fig. 2C and D). However, the treatment with berberine agonist significantly reversed the changes compared with the TBI mice (Fig. 2).

Protective effect of berberine agonist on choline acetyltransferase (ChAT) and acetylcholinesterase (AchE) activity. Compared with the control group, TBI inhibited ChAT activity and induced AchE activity in untreated TBI mice (Fig. 3). Berberine significantly promoted ChAT activity and inhibited AchE activities in treated TBI mice, compared with the TBI model group (Fig. 3).

Protective effect of berberine agonist on MMP-3 and -9 protein expression. Following treatment with berberine agonist, protein expression of MMP-3 and -9 in the TBI model group was increased compared with the control group (Fig. 4). The increase of MMP-3/9 protein expressions in TBI mice was significantly suppressed by berberine agonist treatment (Fig. 4).

Protective effect of berberine agonist on oxidative responses in mice. The effect of berberine agonist on TBI, GSH-PX, GSH and SOD activity, and MDA content was measured. GSH-PX, GSH and SOD activity was significantly inhibited and MDA level was significantly increased in the TBI model group compared with the control group (Fig. 5). Treatment with berberine agonist significantly increased GSH-PX, GSH and SOD activity and reduced the MDA level compared with the TBI mice (Fig. 5). 
A

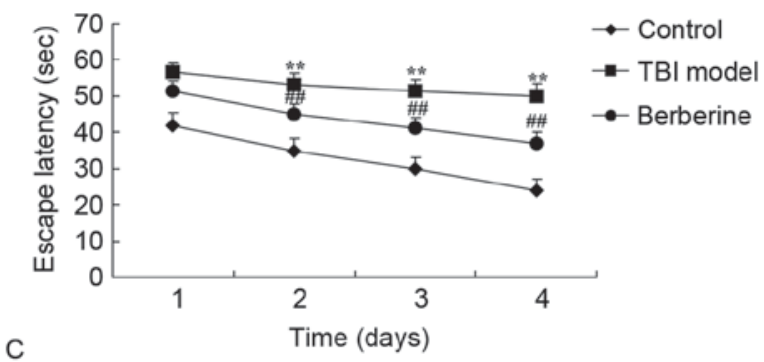

C

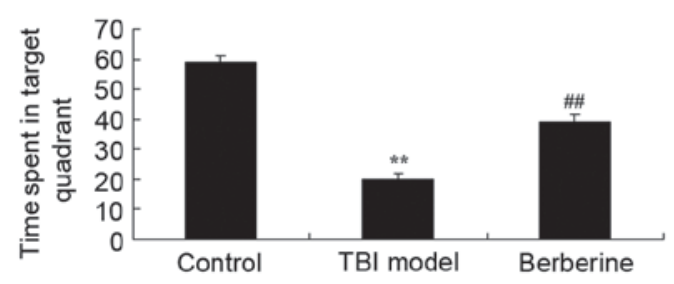

B

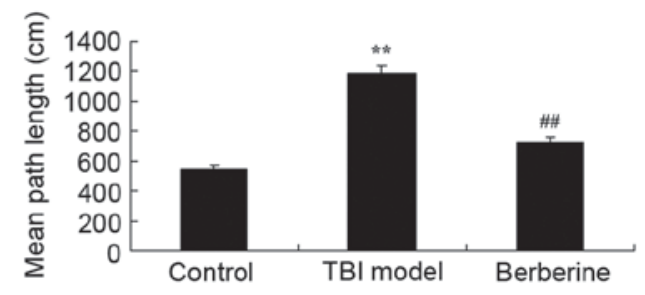

D

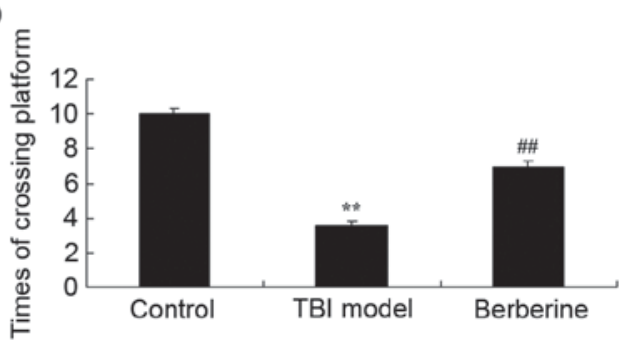

Figure 2. Effect of berberine agonist on Morris water maze test. (A) Effect of berberine agonist on the escape latency. (B) Mean path length. (C) Amount of time to reach the target quadrant. (D) Number of times mice crossed the platform. Data are presented as the mean \pm standard deviation. ${ }^{* *} \mathrm{P}<0.01$ vs. the control group; ${ }^{\#} \mathrm{P}<0.01$ vs. the TBI group. TBI, traumatic brain injury.
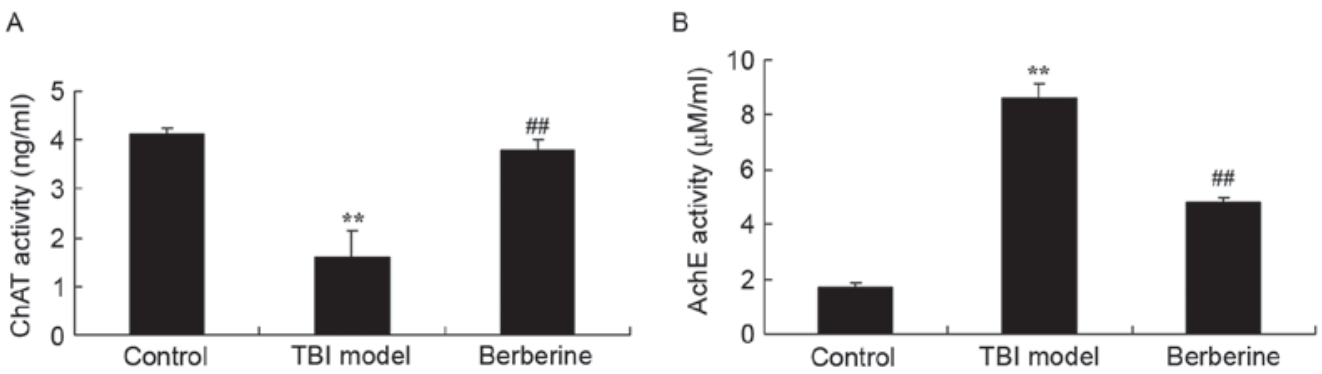

Figure 3. Effect of berberine on ChAT and AchE activity in TBI. (A) Changes in ChAT; and (B) AchE activity. Data are presented as the mean \pm standard deviation. ${ }^{* *} \mathrm{P}<0.01$ vs. the control group; ${ }^{\#} \mathrm{P}<0.01$ vs. the TBI group. ChAT, choline acetyltransferase; AchE, acetylcholinesterase; TBI, traumatic brain injury.

A

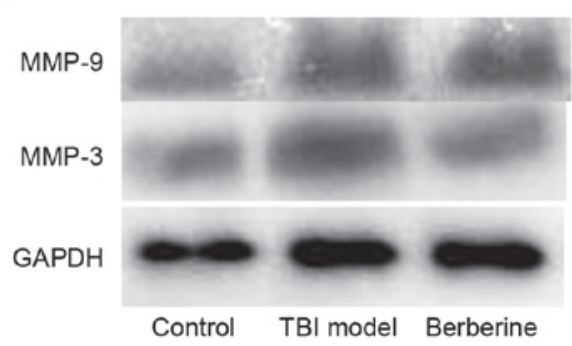

B

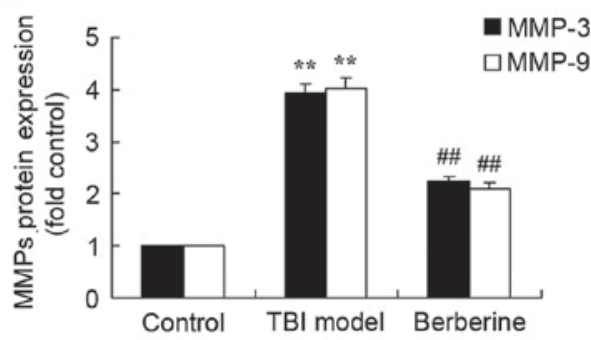

Figure 4. Effect of berberine agonist on MMP-3 and -9 protein expression. (A) Western blotting of MMP-3 and -9. (B) Quantitative analysis of MMP-3 and -9 protein expression. Data are presented as the mean \pm standard deviation. ${ }^{* *} \mathrm{P}<0.01$ vs. the control group; ${ }^{\# \prime} \mathrm{P}<0.01$ vs. the TBI Group. MMP, matrix metalloproteinase; TBI, traumatic brain injury; MMP, matrix metalloproteinase.

Protective effect of berberine on inflammation in mice. To determine whether berberine agonist had an effect on inflammation in TBI, TNF- $\alpha$ and IL- 6 levels were measured. As presented in Fig. 6, the abundance of TNF- $\alpha$ and IL-6 increased in the TBI group compared with the control group. Berberine agonist significantly attenuated TNF- $\alpha$ and IL-6 levels compared with untreated TBI mice (Fig. 6).

Protective effect of berberine agonist on apoptosis levels in $T B I$. To explore the neuroprotective potential of berberine agonist in the treatment of TBI, the anti-apoptotic effects were explored. TBI induced a significant increase of caspase-3 activity and apoptosis regulator BAX (Bax) protein expression, compared with the control group (Fig. 7). Treatment with berberine agonist significantly suppressed caspase-3 activity and Bax protein expression in the treated TBI group compared with the untreated mice with TBI (Fig. 7).

Protective effect of berberine agonist on Sirtl protein expression in TBI. To determine the effect of berberine 


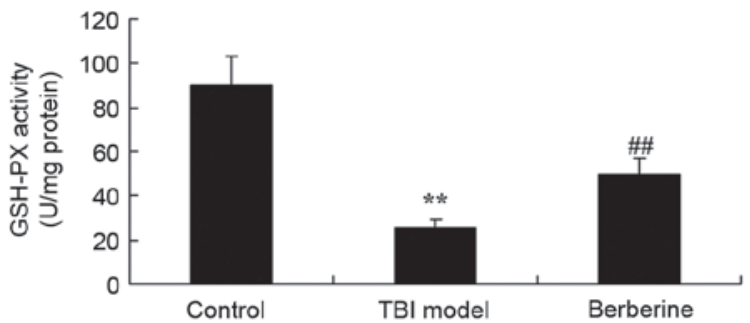

C

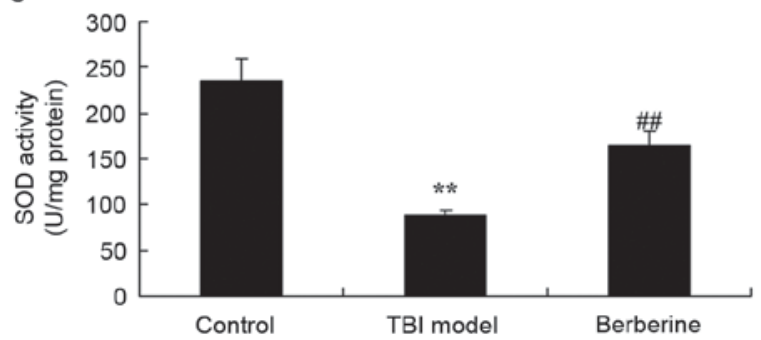

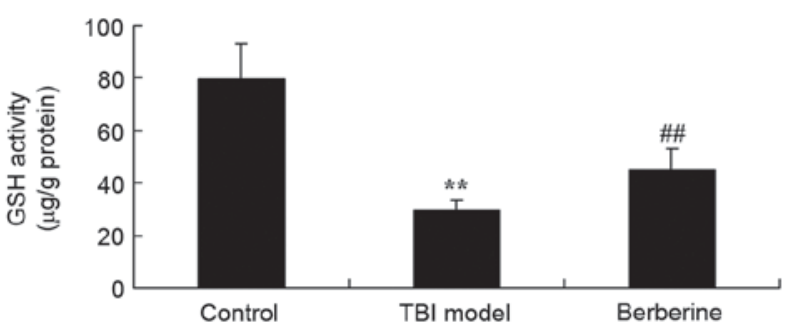

D

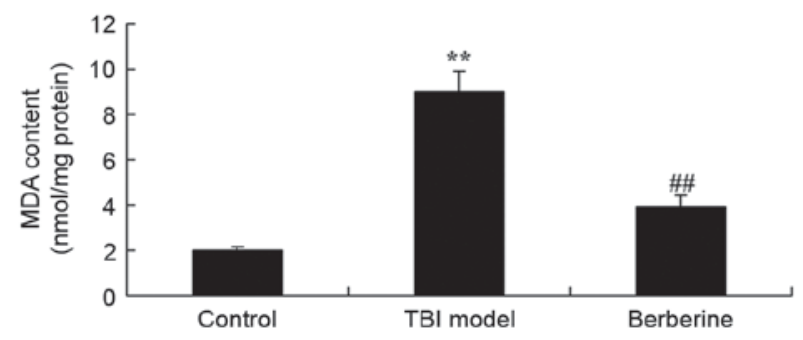

Figure 5. Effect of berberine agonist on oxidative responses. (A) Activity of GSH-PX; (B) GSH; and (C) SOD in mice. (D) MDA levels in mice. Data are presented as the mean \pm standard deviation. ${ }^{* *} \mathrm{P}<0.01$ vs. the control group; ${ }^{\# \#} \mathrm{P}<0.01$ vs. the TBI group. TBI, traumatic brain injury; GSH-PX, glutathione peroxidase 1; GSH, glutathione synthetase; SOD, superoxide dismutase; MDA, malondialdehyde.

A

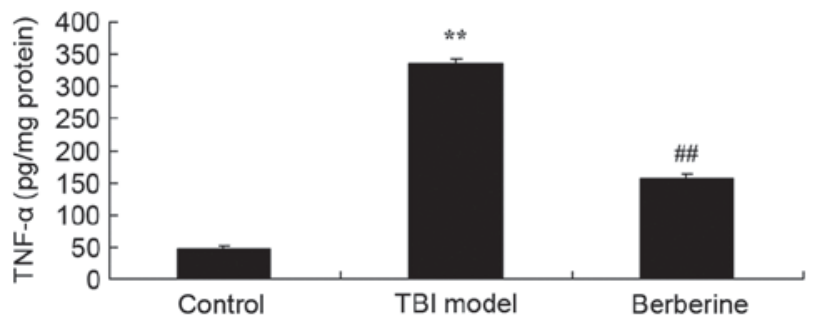

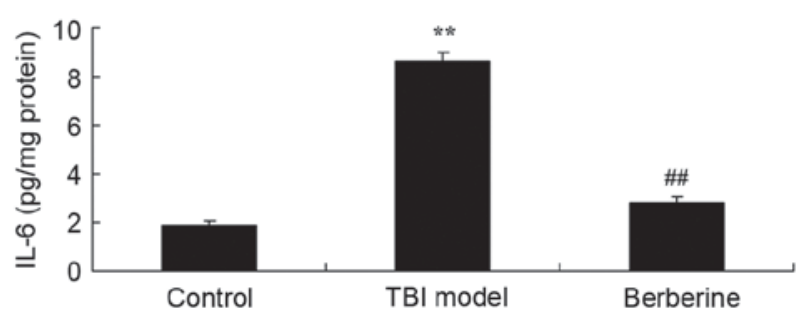

Figure 6. Effect of berberine agonist on inflammation. Levels of (A) TNF- $\alpha$; and (B) IL-6 levels in mice. Data are presented as the mean \pm standard deviation. ${ }^{* *} \mathrm{P}<0.01$ vs. the control group; ${ }^{\# \#} \mathrm{P}<0.01$ vs. the TBI group. TBI, traumatic brain injury; TNF- $\alpha$, tumor necrosis factor- $\alpha$; IL, interleukin.

A

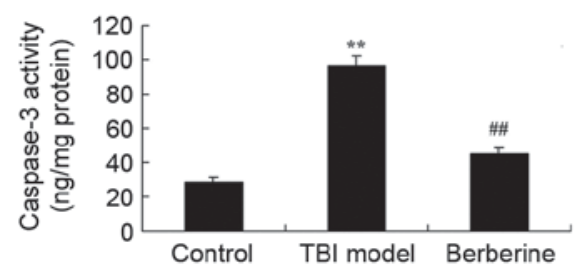

B

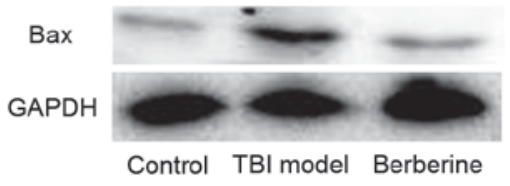

C

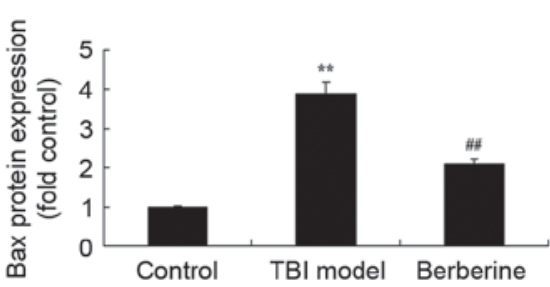

Figure 7. Effect of berberine agonist on apoptosis. (A) Caspase-3 activity in mice. (B) Western blotting assay of Bax protein expression. (C) Quantitative analysis of Bax protein expression in mice. Data are presented as the mean \pm standard deviation. ${ }^{* *} \mathrm{P}<0.01$ vs. the control group; ${ }^{\# \#} \mathrm{P}<0.01$ vs. the TBI group. TBI, traumatic brain injury; Bax, apoptosis regulator BAX.

agonist on Sirt1/FoxO3 $\alpha$ protein expression, Sirt1 and p38 mitogen-activated protein kinase (MAPK) protein expression were measured. The Sirt1 protein expression in the TBI group was lower compared with the control group, and p38 MAPK protein expression in the TBI group was higher than in the control group (Fig. 8). Treatment with berberine agonist significantly induced Sirt1 protein expression and suppressed p38 MAPK protein expression in the treated TBI group compared with the untreated TBI group (Fig. 8).

\section{Discussion}

TBI is a common trauma, with second highest morbidity and highest fatality and disability rates among systemic traumas, making it a prevalent issue in modern society (11). Severe (s)TBI demonstrates high fatality and disability rates, and poor prognosis (12). At present, sTBI is not curable, and the treatment for sTBI remains a challenge for neurosurgeons (13). The results of the present study demonstrated that the neuroprotective effect of berberine agonist rescues learning and memory in severe TBI. 


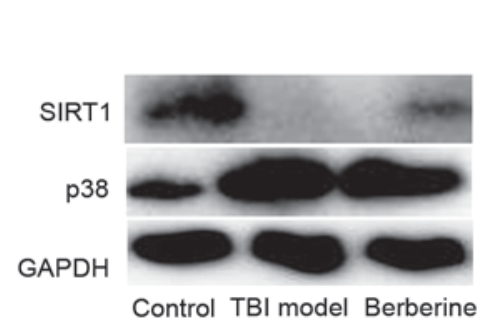

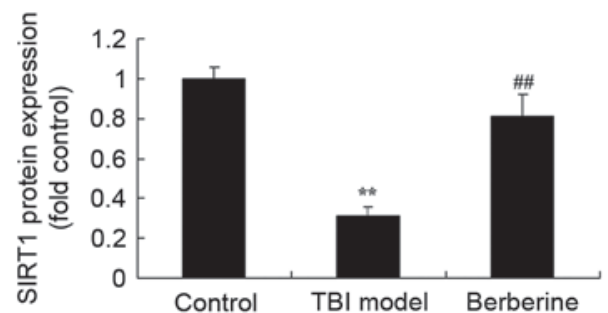

C

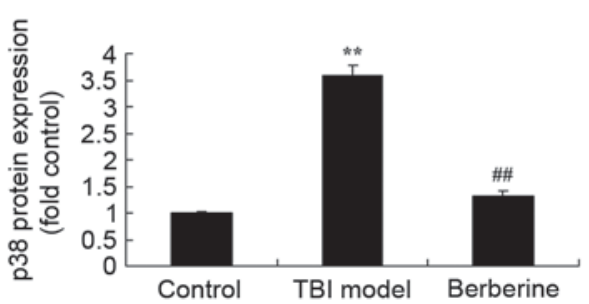

Figure 8. Effect of berberine agonist on Sirt1/p38 MAPK protein expression in mice. (A) Western blot of Sirt1 and p38 MAPK proteins in mice. (B) Quantitative analysis of Sirt1; and (C) p-p38 MAPK protein expression in mice. ${ }^{* *} \mathrm{P}<0.01$ vs. the control group; ${ }^{\#} \mathrm{P}<0.01$ vs. the TBI group. Sirt1, sirtuin-1; MAPK, mitogen-activated protein kinase; TBI, traumatic brain injury.

TBI can be divided into primary and secondary injury stages. The primary injury occurs immediately after injury, is irreversible, and drug interventions are frequently ineffective. The secondary injury induces changes in the intracerebral environment, aggravates the primary disease, and is characterized by long duration and poor prognosis (14). Traditional craniotomy and drug therapy save lives of numerous patients suffering from sTBI, but pathological processes such as secondary cerebral neuron apoptosis and necrosis induced by all kinds of neuron damage factors (including glutamic acid, calcium overload, radical and inflammatory mediators) during the primary injury and perioperative period, cannot be inhibited (15). Efficient reduction of secondary cerebral injury is key to improving the prognosis for TBI patients (15). The mechanism of TBI is complex and involves excessive post-traumatic release of excitatory amino acids, inflammatory response, imbalance of the activity ion channels, hydrocephalus, oxygen radical production and neural death acting simultaneously to trigger a chain reactions, which eventually lead to death of neurons $(6,16)$. In the present study, the protective effect of berberine agonist significantly attenuated inflammation, oxidation and apoptosis levels in severe TBI mice. Yang et al (8) previously demonstrated that berberine exerts an anti-inflammatory role in ocular Behcet's disease.

Post-TBI neural death can be divided into a primary and secondary death. The primary death is referred to as a direct cell death induced by cell membrane disintegration, and the secondary or delayed cell death occurs around and away from the injury sites, and is caused by trauma-induced physiological or biochemical changes in cells (16). Research on the central nervous system cell death remains challenging, as characteristics of dead neurons are different from that of other dead tissue cells even at an ultrastructure level (17). The results of the present study demonstrated the protective effect that Jin et al (10) previously reported, in which Berberine enhances autophagy and mitigates high glucose-induced apoptosis of mouse podocytes through anti-apoptosis effects.

Multiple cells in brain tissue can produce MMPs, including glial cells, microglia, capillary endothelial cells, macrophages and neutrophils (18). MMPs share a common molecular structure of a signal peptide, propeptide and a catalytic domain from the $\mathrm{N}$ - to C-terminal, respectively (10). A predominant function of MMPs is degradation of extracellular matrices, and main components of the basement membrane of blood vessel, including gelatin, type IV and V collagens, cohesin, elastin and fibronectin (10,19). An MMP molecule can directly degrade a certain type or several types of extracellular matrices, or exert its function through the activation of other types of MMPs, initiating a cascade effect (20). MMPs serve important roles in the normal embryonic development and tissue modeling, and in the genesis and development of some diseases including membranous nephropathy, polycystic kidney, atherosclerosis, multiple connective tissue diseases, arthritis and tumor metastasis (21). In the present study, berberine agonist significantly suppressed MMP-3 and -9 proteins in severe TBI mice. Ma et al (10) suggested that berberine inhibits Chlamydophila pneumoniae infection-induced vascular smooth muscle cell migration by downregulating the expression levels of MMP-3 and -9.

Choline acetyltransferase (ChAT) is the generating enzyme of intracerebral neurotransmitter acetylcholine (ACh) involved in learning and memory skills. ChAT abundance in memory-associated cerebral regions is positively correlated with learning and memory abilities (22). ChAT is a marker for cholinergic neurons frequently used to estimate the amount of ACh released by neurons (23). ChAT is synthesized in cholinergic neuron cell bodies and distributed almost parallel to Ach (24). Zhang et al (25) demonstrated that berberine attenuates brain injury in an aluminum-induced rat model of neurodegenerative disease.

In conclusion, the present study demonstrated that the neuroprotective effect of berberine agonist rescues learning and memory skills in severe TBI, through anti-inflammation, anti-oxidation and anti-apoptotic effects. This is, to the best of our knowledge, the first report indicating that berberine agonist may have a neuroprotective effect on learning and memory through regulation of Sirt1/p38 MAPK expression.

\section{Acknowledgements}

Not applicable.

\section{Funding}

No funding was received.

\section{Availability of data and materials}

The datasets used and/or analyzed during the current study are available from the corresponding author on reasonable request. 


\section{Authors' contributions}

JW and YZ contributed to the study concept and design, JW wrote the paper, JW and YZ performed the experiments and JW and YZ revised the article and approved the final version for publication.

\section{Ethics approval and consent to participate}

The present study was approved by the Ethics Committee of Gansu Provincial Hospital (Gansu, China).

\section{Consent for publication}

Not applicable.

\section{Competing interests}

The authors declare no competing interests.

\section{References}

1. Wade SL, Kurowski BG, Kirkwood MW, Zhang N, Cassedy A, Brown TM, Nielsen B, Stancin T and Taylor HG: Online problem-solving therapy after traumatic brain injury: A randomized controlled trial. Pediatrics 135: e487-e495, 2015.

2. Hammond FM, Alexander DN, Cutler AJ, D'Amico S, Doody RS, Sauve W, Zorowitz RD, Davis CS, Shin P, Ledon F, et al: PRISM II: An open-label study to assess effectiveness of dextromethorphan/quinidine for pseudobulbar affect in patients with dementia, stroke or traumatic brain injury. BMC Neurol 16: 89, 2016.

3. Wang X, Ji J, Fen L and Wang A: Effects of dexmedetomidine on cerebral blood flow in critically ill patients with or without traumatic brain injury: A prospective controlled trial. Brain Inj 27: 1617-1622, 2013.

4. Saxena MK, Taylor C, Billot L, Bompoint S, Gowardman J, Roberts JA, Lipman J and Myburgh J: The effect of paracetamol on core body temperature in acute traumatic brain injury: A randomised, controlled clinical trial. PLoS One 10: e0144740, 2015.

5. Du G, Zhao Z, Chen Y, Li Z, Tian Y, Liu Z, Liu B and Song J: Quercetin attenuates neuronal autophagy and apoptosis in rat traumatic brain injury model via activation of PI3K/Akt signaling pathway. Neurol Res: Oct 3, 2016 (Epub ahead of print).

6. Chuang $\mathrm{CH}$, Hsu YC, Wang CC, Hu C and Kuo JR: Cerebral blood flow and apoptosis-associated factor with electroacupuncture in a traumatic brain injury rat model. Acupunct Med 31: 395-403, 2013.

7. Hu ZG, Wang HD, Qiao L, Yan W, Tan QF and Yin HX: The protective effect of the ketogenic diet on traumatic brain injury-induced cell death in juvenile rats. Brain Inj 23: 459-465, 2009.

8. Yang Y, Wang Q, Xie M, Liu P, Qi X, Liu X and Li Z: Berberine exerts an anti-inflammatory role in ocular Behcet's disease. Mol Med Rep 15: 97-102, 2017.

9. Habtemariam S: Berberine and inflammatory bowel disease: A concise review. Pharmacol Res 113: 592-599, 2016.

10. Ma L, Zhang L, Wang B, Wei J, Liu J and Zhang L: Berberine inhibits Chlamydia pneumoniae infection-induced vascular smooth muscle cell migration through downregulating MMP3 and MMP9 via PI3K. Eur J Pharmacol 755: 102-109, 2015.
11. NICE-SUGAR Study Investigators for the Australian and New Zealand Intensive Care Society Clinical Trials Group and the Canadian Critical Care Trials Group; Finfer S, Chittock D, Li Y, Foster D, Dhingra V, Bellomo R, Cook D, Dodek P, Hebert $\mathrm{P}$, et al: Intensive versus conventional glucose control in critically ill patients with traumatic brain injury: Long-term follow-up of a subgroup of patients from the NICE-SUGAR study. Intensive Care Med 41: 1037-1047, 2015.

12. Matuseviciene G, Borg J, Stålnacke BM, Ulfarsson T and de Boussard C: Early intervention for patients at risk for persisting disability after mild traumatic brain injury: A randomized, controlled study. Brain Inj 27: 318-324, 2013.

13. Nägeli M, Fasshauer M, Sommerfeld J, Fendel A, Brandi G and Stover JF: Prolonged continuous intravenous infusion of the dipeptide L-alanine-L-glutamine significantly increases plasma glutamine and alanine without elevating brain glutamate in patients with severe traumatic brain injury. Crit Care 18: R139, 2014.

14. Liu S, Zhang L, Wu Q, Wu Q and Wang T: Chemokine CCL2 induces apoptosis in cortex following traumatic brain injury. J Mol Neurosci 51: 1021-1029, 2013.

15. Ge X, Huang S, Gao H, Han Z, Chen F, Zhang S, Wang Z, Kang C, Jiang R, Yue S, et al: miR-21-5p alleviates leakage of injured brain microvascular endothelial barrier in vitro through suppressing inflammation and apoptosis. Brain Res 1650: 31-40, 2016.

16. Bentz K, Molcanyi M, Schneider A, Riess P, Maegele M, Bosche B, Hampl JA, Hescheler J, Patz S and Schäfer U: Extract derived from rat brains in the acute phase following traumatic brain injury impairs survival of undifferentiated stem cells and induces rapid differentiation of surviving cells. Cell Physiol Biochem 26: 821-830, 2010.

17. Liu SJ, Zou Y, Belegu V, Lv LY, Lin N, Wang TY, McDonald JW, Zhou X, Xia QJ and Wang TH: Co-grafting of neural stem cells with olfactory en sheathing cells promotes neuronal restoration in traumatic brain injury with an anti-inflammatory mechanism. J Neuroinflammation 11: 66, 2014.

18. Reinhard SM, Razak K and Ethell IM: A delicate balance: Role of MMP-9 in brain development and pathophysiology of neurodevelopmental disorders. Front Cell Neurosci 9: 280, 2015.

19. Grossetete M, Phelps J, Arko L, Yonas H and Rosenberg GA: Elevation of matrix metalloproteinases 3 and 9 in cerebrospinal fluid and blood in patients with severe traumatic brain injury. Neurosurgery 65: 702-708, 2009.

20. Wiggins-Dohlvik K, Merriman M, Shaji CA, Alluri H, Grimsley M, Davis ML, Smith RW and Tharakan B: Tumor necrosis factor- $\alpha$ disruption of brain endothelial cell barrier is mediated through matrix metalloproteinase-9. Am J Surg 208: 954-960, 2014.

21. Wang Y, Fan X, Tang T, Fan R, Zhang C, Huang Z, Peng W, Gan P, Xiong $X$, Huang $W$ and Huang $X$ : Rhein and rhubarb similarly protect the blood-brain barrier after experimental traumatic brain injury via gp91phox subunit of NADPH oxidase/ROS/ERK/MMP-9 signaling pathway. Sci Rep 6: 37098, 2016.

22. Dixon CE, Flinn P, Bao J, Venya R and Hayes RL: Nerve growth factor attenuates cholinergic deficits following traumatic brain injury in rats. Exp Neurol 146: 479-490, 1997.

23. Cha Y, Lee SH, Jang SK, Guo H, Ban YH, Park D, Jang GY, Yeon S, Lee JY, Choi EK, et al: A silk peptide fraction restores cognitive function in AF64A-induced Alzheimer disease model rats by increasing expression of choline acetyltransferase gene. Toxicol Appl Pharmacol 314: 48-54, 2017.

24. Pineda RG, Neil J, Dierker D, Smyser CD, Wallendorf M, Kidokoro H, Reynolds LC, Walker S, Rogers C, Mathur AM, et al: Alterations in brain structure and neurodevelopmental outcome in preterm infants hospitalized in different neonatal intensive care unit environments. J Pediatr 164: 52-60. e2, 2014.

25. Zhang J, Yang JQ, He BC, Zhou QX, Yu HR, Tang Y and Liu BZ: Berberine and total base from rhizoma Coptis chinensis attenuate brain injury in an aluminum-induced rat model of neurodegenerative disease. Saudi Med J 30: 760-766, 2009. 